Arts

et Savoirs

\section{Arts et Savoirs}

$14 \mid 2020$

Styles de pensée, pensées du style. Écrire le vivant au XIXe siècle

\title{
Un style « au service de la vérité » : la critique littéraire de la seconde moitié du XIX ${ }^{e}$ siècle
}

A Style "in the Service of Truth". Literary Criticism in the Second Half of the 19th Century

Marine Riguet

\section{OpenEdition \\ Journals}

Édition électronique

URL : http://journals.openedition.org/aes/3430

DOI : 10.4000/aes.3430

ISSN : 2258-093X

Éditeur

Laboratoire LISAA

Référence électronique

Marine Riguet, «Un style « au service de la vérité » : la critique littéraire de la seconde moitié du

XIX ${ }^{e}$ siècle », Arts et Savoirs [En ligne], 14 | 2020, mis en ligne le 29 décembre 2020, consulté le 21

février 2021. URL : http://journals.openedition.org/aes/3430 ; DOI : https://doi.org/10.4000/aes.3430

Ce document a été généré automatiquement le 21 février 2021.

Centre de recherche LISAA (Littératures SAvoirs et Arts) 


\title{
Un style « au service de la vérité »: la critique littéraire de la seconde moitié du XIX ${ }^{\mathrm{e}}$ siècle
}

\author{
A Style "in the Service of Truth". Literary Criticism in the Second Half of the \\ 19th Century
}

Marine Riguet

\section{Introduction}

1 Dans la seconde moitié du XIXe siècle, les sciences du vivant occupent une large part de la scène intellectuelle. Presque simultanément, la publication anglaise de L'Origine des espèces de Darwin (1859), l'Introduction à l'étude de la médecine expérimentale de Claude Bernard (1865), et la controverse sur la génération spontanée entre Pasteur et Pouchet (1860-1865) suscitent des débats sur la notion de vie qui débordent très largement du domaine scientifique, pour venir alimenter les discussions philosophiques, artistiques et littéraires. La critique littéraire s'efforce de se positionner par rapport à ce nouveau champ de savoirs. Elle ne se contente pas de prendre part à une vaste entreprise de vulgarisation, mais elle cherche à questionner et à mettre en acte cette mutation épistémologique, en la réappliquant aux phénomènes littéraires ${ }^{1}$.

Dans ce contexte, le discours scientifique acquiert une prédominance ambiguë, sorte de modèle-rival auquel nombre de critiques font appel sous la forme d'emprunts lexicaux ou notionnels. Mais pour certains, c'est en véritable modèle d'écriture qu'il s'impose. $\mathrm{Au}$ sujet de son ami Claude Bernard, auquel il succède à l'Académie française, Ernest Renan déclare: "Son style, c'est sa pensée elle-même; et comme cette pensée est toujours grande et forte, son style aussi est toujours grand, solide et fort. $»^{2}$ Renan élève le discours scientifique au rang de modèle à suivre pour tout penseur, théoricien ou esthéticien, y compris dans le champ littéraire: «Rhétorique excellente que celle du savant, car elle repose sur la justesse du style vrai, sobre, proportionné à ce qu'il s'agit 
d'exprimer, ou plutôt sur la logique, base unique, base éternelle du bon style. $»^{3}$ Pour comprendre ce qui pourrait nous paraître une curieuse extrapolation, il nous faut resituer le rôle joué par la science dans le débat sur la rhétorique qui anime la critique littéraire de la seconde moitié du siècle.

\section{Pour une a-rhétorique}

3 Tandis que le discours des sciences du vivant occupe une place grandissante dans les programmes universitaires et sous la presse des éditeurs, le procès de la rhétorique celle de l'éloquence classique et des enseignements littéraires - fait rage. Son déclin comme discipline est si connu qu'on pourrait le résumer aussi concisément que le fait Todorov: "Depuis le $\mathrm{xIx}^{\mathrm{e}}$ siècle, la rhétorique classique n'existe plus. ${ }^{4}$ Cette rhétorique classique, c'est l'art de l'éloquence des Grecs qui, plutôt qu'un art du discours, fait figure d'accessoire. Réduite à une valeur ornementale, elle est associée à un souci de plaire en totale contradiction avec toute rigueur scientifique. "Vous avez horreur de la rhétorique, et vous avez bien raison » déclare Renan à Ferdinand de Lesseps, lors de sa réception à l'Académie française en 1885 : «C'est, avec la poétique, la seule erreur des Grecs. Après avoir fait des chefs-d'œuvre, ils crurent pouvoir donner des règles pour en faire : erreur profonde $! »^{5}$ L'argument est on ne peut plus clair. Si la rhétorique règle le style, si elle s'enseigne, elle fait alors obstruction à la justesse de la pensée et à l'expression de la vérité par définition sans fard. Ramenée à la dichotomie beau/vrai, elle s'entend finalement à la fin du siècle de façon tronquée, voire caricaturale, comme une "scolastique littéraire dont le règne est fini $»^{6}$ - soit plus que jamais éloignée de cette rhétorique "au service de la vérité » qui était prescrite par Cicéron, Tacite et Quintilien.

4 Ce procès de la rhétorique ${ }^{7}$, amorcé par les romantiques dès le début du siècle, prend dans les années 1860 un nouveau tournant. Apparenté à la question du style, il est lié à la défense d'une écriture sans ornement, à un idéal du simple qui fait admirer Pascal et Descartes plutôt que les romantiques. Un style, en somme, si proche de la pensée qu'il ne s'enseigne pas. Cette vision prend racine dans le discours prononcé par Buffon à l'Académie française en 1753, et auquel se réfèrent presque unanimement les critiques littéraires du siècle suivant (nous avons pu l'y retrouver chez Antoine Albalat, Jules Barbey d'Aurevilly, Paul Bourget, Ferdinand Brunetière, Émile Deschanel, Émile Faguet, Remy de Gourmont, Jean-Marie Guyau, Alfonse de Lamartine, Gustave Lanson, Désiré Nisard, Armand de Pontmartin, Paul Stapfer et Hippolyte Taine). Deux adages surtout en sont retenus: «le style, c'est l'homme», et «le style n'est que l'ordre et le mouvement qu'on met dans ses pensées $»^{8}$. Autrement dit, le style est redéfini comme un trait singulier propre à chaque auteur, indissociable de sa façon de penser et, de fait, non réductible à des règles collectives. Renan reprend très clairement cet argument dans la suite de son discours à Ferdinand de Lesseps : "Bien parler, c'est penser tout haut. Le succès oratoire et littéraire n'a jamais qu'une cause, l'absolue sincérité. $»^{9}$ Or, face à cette "absolue sincérité ", il est évident que la rhétorique fait figure d'artifice, d'effort anti-naturel et qui plus est inhibant. Elle devient l'ennemi du style, et même, par extension, de la singularité de l'écrivain. Cette dévalorisation gagne l'imaginaire collectif, au point qu'on en trouve des représentations jusque dans les romans : dans L'Immortel, par exemple, Alphonse Daudet ne se prive pas de fustiger le professeur de rhétorique sous les traits d'un censeur brimant l'originalité et le génie: «le vieux 
s'acharnait des outils et des ongles, arrivait à nous faire tous propres et plats comme un banc d'école $»^{10}$. La rhétorique, alors réduite à un exercice formaliste suranné, souffre du côté des écrivains d'un véritable discrédit ${ }^{11}$.

À travers cette dissociation du style littéraire et de la rhétorique, il est intéressant de noter, chez nombre de critiques, le besoin de mettre en scène une rupture entre des pratiques classiques révolues, propres aux belles-lettres, et l'époque dite moderne. Alors que les romantiques souhaitaient réformer la rhétorique classique en la centrant sur la sensibilité et l'imagination ${ }^{12}$, le dernier tiers du siècle préfère la rejeter sans concession. La littérature se débarrasse en quelque sorte d'une tradition littéraire sclérosante et devenue inapte à la nourrir. Et puisqu'elle n'est plus capable de trouver à l'intérieur de son champ ses propres outils, elle doit les puiser au-dehors. C'est dans ce contexte que Buffon devient la première référence citée par la critique littéraire en matière de style, que Désiré Nisard reconnaît en Descartes son plus grand modèle, et qu'Émile Zola apparente son écriture à celle de Claude Bernard. L'homme de science, mieux que le professeur de rhétorique, participe à l'élaboration théorique du style littéraire. Mais derrière la défense d'une écriture claire et efficace se joue surtout une conception nouvelle du langage. Ce que le savant permet de figurer et que la rhétorique traditionnelle était impuissante à rendre, c'est l'idée d'une écriture qui ne chercherait plus à plaire ni à persuader, mais dont la valeur serait contenue dans la pensée même qu'elle traduit ; qui aurait acquis, en bref, l'intransitivité de la vérité. Le remplacement de la rhétorique classique par le modèle scientifique peut ainsi être compris, sur le plan stylistique, comme le moyen détourné de renouer avec l'idéal ancien d'une expression " enrôlée au service de la vérité $~^{13}$. En venant suppléer un héritage littéraire tombé en disgrâce, la science semble la mieux placée pour occuper les fonctions de la rhétorique, précisément parce qu'elle bénéficie d'un statut a-rhétorique.

6 Il va sans dire que ce discrédit général de la rhétorique traditionnelle trouve quelques opposants, rares mais farouches. Brunetière, non des moindres, entre dans la polémique avec un chapitre entier consacré à l'« apologie pour la rhétorique ", qu'il ouvre sentencieusement: "S'il est, comme on l'a dit, des morts qu'il faut qu'on tue, n'en est-il pas peut-être aussi, de loin en loin, qu'il faut qu'on ressuscite, ou dont on essaye de ranimer et de renouveler la mémoire? ${ }^{14}$ Le critique tente ensuite de réhabiliter une acception antique de la rhétorique, qu'il situe par-delà le style, dans l'écriture même :

La rhétorique est l'ensemble des règles et des lois qui gouvernent l'art d'écrire, considéré lui-même comme inséparable de l'art de penser; et, qu'on le sache ou non, - mais je crains qu'on ne le sache fort bien, - ce que l'on nie quand on attaque la rhétorique, c'est qu'il y ait un art de penser et d'écrire. ${ }^{15}$

7 Albalat prend quant à lui le contre-pied de Renan, en considérant que la rhétorique met en valeur les originalités :

Nous avons prouvé par des exemples, nombreux et faciles à multiplier, qu'on trouve non seulement traces de rhétorique chez les écrivains les plus accomplis, mais que cette rhétorique est le fondement même de leur talent. Les procédés de rhétorique de Cicéron, ceux de Montaigne, Bossuet, Montesquieu, Rousseau, tous ces modes d'écrire, visibles et analysables, n'empêchent pas ces auteurs d'être de grands écrivains. Pourquoi ? Parce que l'emploi de ces procédés n'est pas suspect et se dissimule sous la force de la pensée. ${ }^{16}$

8 Mais ces opérations de sauvetage restent vaines. Après la première offensive contre l'enseignement de la rhétorique lancée par Jules Simon en $1872^{17}$, le plan d'études 
prescrit par arrêté ministériel le 22 janvier 1885 et la réforme de 1890 chassent peu à peu la rhétorique des programmes du secondaire. Contre des exercices de style et de discours jugés dépassés, le parti moderne préfère une culture des idées procédant par philosophie et méthode. Les bases du programme sont jetées sans ambages dans les Instructions de 1890 :

Il ne s'agit pas de développer les facultés de l'esprit, au sens le plus étroit du mot ; de créer une habileté à discuter, à composer, à tourner agréablement des vers et de la prose; ou du moins il ne s'agit de tout cela que très secondairement. [...] l'enseignement sera moins littéraire, mais plus philosophique et plus humain; il deviendra à sa manière une véritable leçon de choses morales professée par des écrivains de génie. ${ }^{18}$

La lutte contre la rhétorique symbolise le besoin de quitter la forme pour la substance ; et c'est Lanson, comme on le sait, qui l'achèvera en assénant le coup de grâce.

Nous atteignons ici le second aspect de la polémique ${ }^{19}$. Car la rhétorique n'est pas seulement mise en accusation par rapport à la notion de style littéraire; elle l'est, plus foncièrement, en tant que discipline critique. Pour Lanson, qui commence sa carrière comme professeur de rhétorique avant d'être nommé à la tête de l'École Normale, ce sont les humanités qui sont en péril. Parce qu'elles "se rapetissent à la rhétorique ", elles n'offrent même plus "un art de penser», mais "un art de parler bien sans penser $\aleph^{20}$. Or rééduquer les esprits, réapprendre à penser, implique de réfléchir à partir de la littérature, c'est-à-dire des textes mêmes, plutôt que par le biais d'axiomes et d'académismes sclérosés.

Il n'y aura de menacée que la rhétorique, et cette malheureuse habitude de ne pas examiner la vérité des choses, sous le prétexte d'en analyser ou admirer la beauté, ce que l'on appelle la culture du goût littéraire, et qui n'en est que la perversion et l'abus. $^{21}$

11 Si l'esprit littéraire est en bout de course à cause de l'enseignement traditionnel, il faut donc le réformer. Il ne fait aucun doute, pour Lanson, que la rhétorique a fait son temps, et qu'elle doit laisser sa place à des méthodes nouvelles: "Les humanités se renouvelleront, libérées de la rhétorique, et dirigées par le souci de la formation scientifique de l'esprit. $\rangle^{22}$ À nouveau, la rupture avec l'héritage littéraire amène à se tourner vers le discours scientifique. C'est que «l'éducation scientifique, seule, peut améliorer toute la jeunesse d'une nation et lui donner l'esprit de précision, de méthode et de discipline nécessaire aux œuvres collectives. $»^{23}$ L'argument est ici presque démocratique. La formation scientifique participe à l'éducation sociale et civique des étudiants; elle véhicule en somme les grandes valeurs républicaines, à savoir la vérité et la solidarité, contre l'individualisme de l'ancienne éducation littéraire. Dans sa conférence sur la méthode qu'il tient à Bruxelles en 1909, Lanson le réaffirme avec plus de pugnacité :

La critique, dogmatique, fantaisiste ou passionnée, divise; l'histoire littéraire réunit, comme la science dont l'esprit s'inspire; elle devient ainsi un moyen de rapprochement entre des compatriotes que tout le reste sépare et oppose, et c'est pourquoi j'oserais dire que nous ne travaillons pas seulement pour l'érudition, ni pour l'humanité; nous travaillons pour nos patries. ${ }^{24}$

12 En se donnant pour tâche de réformer l'éducation, la III $^{\mathrm{e}}$ République fait de la rhétorique le symbole de l'ennemi, soit un vieil enseignement hérité des jésuites, élististe et antidémocratique. En plus de la mise en scène d'une fracture entre anciens 
et modernes, le pas pris par l'esprit scientifique sur l'esprit littéraire porte la marque d'un enrôlement qui confine à la mythologie républicaine.

La nature scientifique de l'étude littéraire ne tient pas pour Lanson à l'imitation d'une forme, d'une méthode. Il est plutôt question de réintroduire "l'esprit scientifique » au sein du domaine littéraire, de façon à acquérir une dimension non pas rhétorique mais cognitive du discours. « Notre manière de participer à la vie scientifique, affirme-t-il encore, la seule qui ne trompe pas, c'est de développer en nous l'esprit scientifique. $»^{25}$ Ce qu'entend Lanson dépasse en fait le cadre des sciences; il nomme "attitude scientifique universelle $»^{26}$ une approche rationnelle, attachée à la compréhension du réel, et apte à produire la connaissance. Aussi la critique littéraire doit-elle être scientifique en partant des règles que lui dictent ses propres objets, et non pas ceux de la biologie ou de la médecine. C'est la raison pour laquelle, en opposant une formation scientifique de la littérature à la rhétorique classique, Lanson se fait l'apôtre de l'explication de texte dans l'enseignement secondaire, et de l'histoire littéraire dans le supérieur. La connaissance de la littérature ne peut naître qu'à partir d'elle-même, et de l'étude la plus impartiale possible de ses objets. Quant à la critique, elle ne peut plus subsister que sous les traits de l'histoire littéraire, hors de tout impressionnisme ou dogmatisme, mais avec le recul qu'elle peut acquérir sur les textes pris comme documents :

Notre métier consiste à séparer partout les éléments subjectifs de la connaissance objective, l'impression esthétique des passions et des croyances partiales, à éliminer tout ce qui ne peut être productif que d'erreur ou d'arbitraire, à retenir, filtrer, évaluer tout ce qui peut concourir à former une représentation exacte du génie d'un écrivain ou de l'âme d'une époque. ${ }^{27}$

Avec Lanson, donc, la République sonne le glas de la rhétorique. L'enseignement de la rhétorique est définitivement effacé des programmes du secondaire par la grande réforme de l'enseignement de 1902. Ce bref rappel de la polémique met bien en avant l'opposition qui est construite à l'époque entre, d'une part, une tradition rhétorique dépassée et, d'autre part, l'approche scientifique avec laquelle on souhaite aborder la littérature. Au fond, ce qui s'amorce n'est guère l'avènement d'une a-rhétorique, voire d'une "écriture blanche ${ }^{28}$ " dont le style, minimaliste, se réduirait à la vérité de l'énoncé ; mais plutôt l'abandon de la rhétorique et de l'éloquence classiques au profit d'une rhétorique épistémique, chargée de faire savoir.

\section{Entre la robe et la blouse}

Dans ce contexte, les auteurs de la seconde moitié du XIX ${ }^{e}$ siècle apporteront des réponses hétérogènes et singulières, si bien qu'il est impossible de les regrouper en faisant du "style scientifique » un critère de classification. Ce qui nous intéresse en revanche, c'est cette intention de produire un discours de nature épistémique, telle qu'elle est exprimée de Jean-Jacques Ampère ${ }^{29}$ à Lanson, et qui pousse nombre d'entre eux à recourir à une rhétorique des sciences. Nous nous situons à cet égard dans le sillage des travaux de Jacky Martin, en entendant la rhétorique au sens large, comme " toute stratégie de discours orientée vers une certaine finalité ${ }^{30}$ » - cette finalité étant, dans le contexte scientifique, l'acceptation de l'énoncé par une communauté de spécialistes authentifiant sa validité et l'intégrant à un domaine de référence collectif. En postulant à la fois une dimension cognitive (phénomènes d'objectivation et de rationalisation) et rhétorique (phénomènes de légitimation et de valorisation), Jacky 
Martin concède au discours dit scientifique un statut qu'il qualifie d'« hybride ", à michemin entre la linguistique et la sociologie. Celui-ci, par sa mise en tension entre un régime assertif et un régime modalisé, programme à la fois la validation de l'énoncé et sa possible réappropriation. De cette façon, le discours ne fait pas seulement acte de communication ; il produit à proprement parler le savoir.

Et c'est bien cette ambition de produire le savoir qui est partagée par une majorité de critiques littéraires. Loin du jugement de goût, ils entendent faire de la critique une nouvelle science esthétique aux mains d'experts. Cette ambition se comprend en partie par l'influence de l'esthétique allemande du XVIII ${ }^{e}$ siècle, reçue en France tardivement. $\mathrm{Au}$ milieu du XIX siècle, les premières traductions faites par Charles Bénard de l'Esthétique d'Hegel et de Schelling ${ }^{31}$, ainsi que celle réalisée par Jules Barni de la Critique du jugement de Kant ${ }^{32}$, amorcent une réception de l'esthétique, entendue en Allemagne, depuis Baumgarten, comme "science de la connaissance sensible ${ }^{33}$. Quelques années plus tard, La Science du beau de Charles Lévêque ${ }^{34}$, publié en 1861 et récompensé par l'Académie des sciences morales et politiques, entérine une esthétique française qui doit, d'une part, épouser le caractère d'une science objective et, de l'autre, puiser dans la psychologie les moyens de son analyse. Cette émergence de l'esthétique comme discipline philosophique pose deux enjeux à la critique littéraire : se réapproprier la question du beau face à la philosophie et en faire un objet de connaissance scientifique. Nombre de critiques y voit aussi l'occasion de détacher définitivement la pratique critique de son enracinement métaphysique et de ses accents idéalistes. Pour marquer ce détachement, ils se tournent spontanément vers les sciences du vivant, modèles du discours d'autorité de l'époque. «Quand la biologie sera constituée, c'est-à-dire dans quelques millions d'années, on pourra peut-être construire une sociologie [...]; après quoi, il sera loisible de créer sur des bases solides une science esthétique $»^{35}$ diagnostique Anatole France. Sur ce parti pris, Émile Hennequin préfère écarter d'emblée l'ancienne critique et forger, derrière le terme d'esthopsychologie, une critique esthétique scientifique. Pensée comme science, débarrassée de sa fausse prétention à juger le beau, cette dernière cesse d'être un discours esthétique au sens métaphysique pour entrer dans la sphère de la connaissance empirique. Plutôt que de former au goût, elle hisse la "science du beau " à son rang le plus élevé, c'est-à-dire à l'étude des émotions esthétiques grâce aux moyens fournis par les sciences modernes - la physiologie, la psychologie et la sociologie expérimentales.

17 La plupart des critiques choisissent ainsi de marquer une césure : la nouvelle esthétique fournie par la critique littéraire entend refonder une théorie du beau construite, non sur la perception subjective, mais sur des analyses rigoureuses, des principes et des critères suffisamment objectifs pour avoir valeur de général. Contrairement à l'esthétique classique, déterminée en amont par une autocratie, elle acquiert sa pertinence sur les méthodes empiriques des sciences. Taine, élu à la toute jeune chaire d'esthétique de l'École des Beaux-Arts en 1864, est peut-être celui qui invite avec le plus de force à reconsidérer l'approche critique selon un protocole scientifique. Avec lui, la rupture est consommée. Et dans la visée de cette nouvelle esthétique s'engagent tour à tour les théoriciens qui cherchent à jeter les bases d'une critique moderne, tels Renan, Hennequin, Bourget, ou encore Brunetière, que nous citons ici :

C'est que nous avons beau faire, nous pouvons bien méconnaître la nature des choses, et la nier au besoin - mais nous ne pouvons pas la détruire. L'ancienne esthétique ou l'ancienne critique « donnait d'abord la définition du beau », nous dit quelque part M. Taine ; et, partant de là, « elle absolvait, condamnait, admonestait 
et guidait ». Mais tout le tort qu'elle avait, vous l'avez vu - et je pense qu'il est assez considérable, - c'était de commencer par la fin, et de poser en principe une définition du beau que son objet même est de rechercher. Les définitions sont le terme de la science, elles n'en sont point le début. Mais qu'elles en demeurent l'objet, c'est de quoi M. Taine a dû s'apercevoir quand, chargé d'enseigner l'esthétique à l'école des Beaux-Arts, il s'est aperçu que la sculpture grecque, la peinture hollandaise et la peinture italienne avaient un autre intérêt, plus profond et plus actuel, que de refléter pour nous l'état d'âme d'un contemporain de Barneveldt, de Léon $\mathrm{X}$ ou de Périclès. Lui aussi, il a bien fallu qu'il donnât sa "définition du beau »; il a bien fallu qu'il cherchât un principe de distinction et de classification des œuvres; il a bien fallu qu'il se fit enfin un criterium - si j'ose, en parlant de lui, me servir de cette expression, - et comme il est, d'ailleurs, le plus consciencieux des hommes, il en a pris son parti ; et il s'en est fait un, dans ses leçons sur l'Idéal dans l'art. Vous y trouverez le complément de son esthétique, et pour ainsi parler, le couronnement de son système, puisque depuis lors, vous le savez, il s'est détourné de la critique pour s'appliquer uniquement à l'histoire. ${ }^{36}$

Autrement dit, le nouveau critique quitte l'autorité du juge éclairé, du censeur du «bon goût», pour revêtir celle du chercheur de laboratoire. S'il gagne sa légitimité en matière d'esthétique, c'est en bâtissant sa faculté de juger sur l'examen du beau, de sa nature et de ses conditions. «La vraie esthétique suppose la science. Le savant seul a le droit d'admirer ${ }^{37} »$ reprend Renan dans la table analytique de L'Avenir de la science, qu'il écrit, rappelons-le, dans ses années de jeunesse, avant même que La Science du beau de Lévêque ait fait grand bruit.

19 En fait, avec la défense d'une rhétorique «scientifique », c'est la défense du jugement critique qui est menée. Contrairement aux idées reçues, l'influence scientifique de la nouvelle critique n'efface pas toute ambition de juger. Rappelons que le premier rôle du critique, pour Sainte-Beuve, est encore de distinguer la vraie littérature d'une fausse, qu'il qualifie d'« industrielle ", prolongeant ainsi la voie ouverte par Ampère :

Cette critique, Messieurs, a un double objet ; l'importance et le mérite des ouvrages qu'elle évoque à son tribunal. Au milieu de cette foule de productions qu'entassent les siècles, le premier devoir de la critique est de distinguer celles qui sont dignes de prendre place dans l'histoire, de fixer le rang qu'elles y doivent tenir. ${ }^{38}$

Wolf Lepenies, au cours de son étude sur Sainte-Beuve, ne manque d'ailleurs pas de souligner l'importance tenue par le vocabulaire judiciaire emprunté au droit et aux tribunaux dans l'œuvre du critique ${ }^{39}$. «Insisterai-je ici sur l'obligation de juger? rappellerai-je qu'elle est comme impliquée dans l'étymologie même du nom de la critique $?^{40}$ " clame encore Brunetière. Durant la seconde moitié du siècle, le jugement se trouve surtout au cœur d'une réélaboration afin d'être à la fois désinvesti des critères classiques et réintégré dans une dimension épistémique. Il va s'agir d'élaborer les lois permettant au critique de formuler a posteriori une sorte de jugement d'autorité, juste et par là consensuel. En somme, un jugement de tribunal plutôt que de moraliste. C'est ainsi que nous pouvons par exemple comprendre la prescription faite par Brunetière d'une nouvelle critique dans la lignée de Sainte-Beuve :

Mais s'il y a des familles d'esprits, s'il y a des genres, des espèces dans ces genres, des rangs dans ces espèces, nos impressions ne sont donc plus rien en critique, ni même nos jugements? Que l'on blâme ou qu'on loue, que l'on approuve ou que l'on condamne, ce n'est pas la sentence qui importe; ce n'est pas même le juge; les " considérants » sont tout; et la valeur de ces "considérants ", d'où dépend-elle à son tour, sinon de la connaissance que nous avons - ou que nous pouvons avoir des lois qui gouvernent l'esprit humain ? ${ }^{41}$ 
Une telle mise à l'écart du jugement a priori permet de se prémunir d'une critique d'humeur - non pas pour en faire une critique dénuée d'affects, mais justement pour assurer les assises d'une esthétique «élev[ée] au-dessus de nos goûts »"

\section{La valeur de vérité}

Dans cette perspective, on retrouve chez un grand nombre de critiques littéraires de la seconde moitié du XIx ${ }^{e}$ siècle un effort de rationalisation et de légitimation du discours, répondant à cette double dimension cognitive et rhétorique de la production scientifique. Selon la terminologie de Jacques Fontanille, la visée épistémique d'un discours passe d'abord par une logique de la cognition, propre à ordonner les objets cognitifs sur le schéma de la découverte :

[...] la rationalité cognitive est celle de l'appréhension et de la découverte: appréhension et découverte de la présence du monde et de la présence à soi-même, découverte de la vérité, découverte des liens qui peuvent apparaître entre les connaissances existantes, etc. ${ }^{43}$

En d'autres termes, la logique cognitive assure le passage de l'ignorance à la connaissance. Elle appartient au champ du savoir dans la mesure où elle met en relation les objets cognitifs entre eux, sans avoir besoin de passer par une instance. Ainsi établit-elle l'objectivité et la rationalité du discours scientifique. Ce dernier ne peut cependant se contenter d'énoncer : il doit encore garantir sa valeur véridictoire. À la logique de la découverte s'ajoute donc une logique de la preuve. Comme le souligne Jean-Michel Berthelot, il ne suffit pas au discours d'«apporter des connaissances nouvelles », il lui faut « faire la preuve de leur validité $»^{44}$. Cette seconde caractéristique est peut-être, pour Jean-Michel Berthelot, plus importante encore, au point d'assigner à tout texte scientifique une « vocation probatoire systématique ». Elle est, à proprement parler, la dimension rhétorique du discours scientifique.

Plusieurs ressorts du discours de critique littéraire obéissent à cette logique de la découverte et de la preuve. La prolifération de méthodes critiques et de références textuelles puisées dans les sciences du vivant participe explicitement d'une rationalisation du discours littéraire, en rupture avec les pratiques de la rhétorique classique. Dès le milieu du siècle, Sainte-Beuve prône une "méthode vivante et naturelle substituée aux formules didactiques ${ }^{45} \%$. Par là, il entend rassembler les différents projets d'une critique nouvelle sous l'égide de Bacon, Lamarck et Buffon ; une critique fondée sur l'observation, à l'instar du naturaliste qui observe les êtres de façon à déterminer par induction leurs grandes caractéristiques, puis à les classer par espèces. Analysés suivant cette méthode (observation et raisonnement par induction), les faits littéraires entrent dans un discours de la découverte et de la preuve qui assure leur valeur épistémologique : ils bénéficient à la fois d'une construction méthodique rationalisante, et d'une validation sous autorité scientifique. Sainte-Beuve vient à en faire la profession de foi d'une génération, en reconnaissant chez ses pairs - Renan, Taine, Émile Deschanel - ce même soin à inscrire le discours critique sur un versant épistémique :

Nous tous, partisans de la méthode naturelle en littérature et qui l'appliquons chacun selon notre mesure à des degrés différents, nous tous, artisans et serviteurs d'une même science que nous cherchons à rendre aussi exacte que possible, sans nous payer de notions vagues et de vains mots, continuons donc d'observer sans 
relâche, d'étudier et de pénétrer les conditions des œuvres diversement remarquables et l'infinie variété des formes de talent. ${ }^{46}$

Ce souci de méthode est effectivement l'affaire de toute une nouvelle critique. Citons encore l'histoire évolutionniste des genres littéraires que Brunetière bâtit sur la méthode et les lois de l'histoire naturelle ${ }^{47}$; la récupération de la méthode expérimentale de Claude Bernard par Renan, Zola, Bourget ${ }^{48}$; ou la Méthode scientifique de l'histoire littéraire signée par Georges Renard en 1900. Quelle que soit la singularité de chaque projet critique, l'enjeu est, chaque fois, d'assurer la dimension cognitive du discours littéraire de manière à le faire entrer dans le champ de la connaissance.

Cette stratégie probatoire vient ainsi suppléer le registre judiciaire traditionnel de la critique littéraire. L'invocation d'une méthode en rupture avec la rhétorique classique fait passer le jugement d'une dimension juridique à une dimension scientifique, et accompagne la transition d'une critique de goût vers une critique de savoir. Le changement est axiologique : l'évaluation devient véridictoire avant d'être esthétique ou éthique. En recourant à un discours à valeur de vérité scientifique, la critique tend à intégrer un système culturel, relatif et contingent, au sein d'un système scientifique plus large, universel et permanent. Elle compte ainsi s'assurer un discours sur la littérature pérenne, indépendant du système de valeurs propre au critique lui-même ou à la société qui est la sienne. La vérité est mise en scène comme valeur collective, nécessairement consensuelle, et rationalisante. Brunetière, au détour d'une étude sur Voltaire, en donne la formule: «il en est du jugement littéraire comme de la vérité scientifique, dont la certitude, une fois acquise et démontrée, ne s'accroît point par le nombre des vérifications qu'on en fait ${ }^{49}{ }^{\prime}$. Pour le critique historien comme pour le critique juge, le but n'est pas esthétique ou éthique, mais véridictoire. Le discours judiciaire se fond ainsi dans le discours scientifique afin de partager une même vérité non plus bâtie par des lois humaines mais directement reçue de l'ordre naturel. On se rappelle également ces mots avec lesquels Renard ouvre sa Méthode :

$\mathrm{Et}$, en effet, où est-il l'historien qui oserait déclarer aujourd'hui que la recherche du vrai n'est pas sa première et essentielle préoccupation? Qu'il s'agisse de l'évolution politique, religieuse ou littéraire d'une nation, l'idéal pour quiconque veut la retracer est d'aboutir à des résultats certains et définitifs. ${ }^{50}$

Cette stratégie permet d'affirmer la validité et l'autorité du discours de la critique littéraire en tant que discipline autonome, émancipée des belles-lettres ou de la philosophie, et apte à produire son propre savoir. Mais sa portée est plus large encore. Elle étend cette légitimation à la littérature, qui ne reçoit non plus ses valeurs d'une société, d'une politique ou d'une religion, d'une nécessité hors d'elle, mais d'une vérité qui fait sens par et pour elle-même.

Bien qu'elle adopte une rhétorique épistémique, la critique ne prétend pas objectiver le fait littéraire : au contraire, elle entend légitimer son étude du sujet, du singulier, de l'humain, en le faisant entrer dans un régime de vérité. Ce point n'est pas des moindres, car il permet de lever l'apparente répartition binaire d'une critique positivisteobjective d'un côté, et impressionniste-subjective de l'autre. Chez les uns comme chez les autres, même quand il s'agit de considérer l'œuvre selon des lois scientifiques, l'esthétique sert de ressort à l'affirmation d'une subjectivité constitutive de la relation à la littérature ${ }^{51}$. Ceci permet également de comprendre le rôle prééminent des sciences du vivant dans cette affiliation au modèle scientifique. Car c'est en grande majorité à la physiologie, à la biologie et à la médecine que la critique de la seconde moitié du siècle se réfère. Hisser ses objets littéraires à la hauteur des objets du vivant lui permet, par 
un jeu d'analogies, de leur conférer une même autonomie. La littérature, comme tout être vivant, devient une entité avec ses lois propres, ses origines, son histoire. En somme, une littérature comme "monde à part, détaché des contingences de la réalité $"^{52}$, aussi vraie que la vie même. En ce sens, la rhétorique épistémique de la critique littéraire sert à valoriser la littérature. En la faisant entrer dans le champ de la connaissance, elle affirme son existence et sa nouvelle intransitivité. Nous rejoignons par là cette conclusion de Foucault qui, dans Les Mots et les choses, situe au XIX ${ }^{\mathrm{e}}$ siècle "l'apparition de la littérature", mais "de la littérature comme telle", qui "se distingue de plus en plus du discours d'idées, et s'enferme dans une intransitivité radicale $\aleph^{53}$. La critique littéraire, par sa rupture avec la rhétorique classique et son affirmation comme discours producteur de savoir, prend en charge la naissance de l'idée moderne de littérature.

\section{NOTES}

1. Nous avons développé cela dans notre ouvrage Faire littérature: genèse d'un laboratoire, Paris, Hermann, 2019.

2. Ernest Renan, «Discours de réception à l'Académie française » [3 avril 1879], dans Discours et conférences, Paris, Calmann-Lévy, 1887, p. 14.

3. Ibid.

4. Tzvetan Todorov, Théorie du symbole, Paris, Seuil, « Poétique », 1977, p. 85.

5. Ernest Renan, Discours et conférences, op. cit., p. 130.

6. L'abbé Joseph Bourlier consacre un discours au sujet à l'occasion d'une distribution des prix, cf. Du rôle actuel de la rhétorique dans l'éducation littéraire, L'Union typographique, 1889.

7. Nous ne pouvons restituer ici la polémique de façon exhaustive, mais pour un aperçu plus global du sort de la rhétorique à cette époque, nous renvoyons au chapitre d'Antoine Compagnon «La rhétorique à la fin du XIxe siècle (1875-1900)», dans Marc Fumaroli (dir.), Histoire de la rhétorique dans l'Europe moderne, Paris, PUF, 1999, p. 1215-1260.

8. G. L. Leclerc de Buffon, Discours sur le style, prononcé lors de sa réception à l'Académie française en 1753.

9. Ernest Renan, Discours et conférences, op. cit., p. 131.

10. Alphonse Daudet, L'Immortel, 1888 ; cité par Antoine Compagnon, op. cit., p. 1232.

11. Il n'en reste pas moins que la rhétorique s'enseigne encore. Elle garde qui plus est bonne presse dans la sphère religieuse, en étant affiliée à cette grande éloquence du clergé dont Bossuet et Bourdaloue demeurent les modèles.

12. Voir l'article de Jean Molino, "Quelques hypothèses sur la rhétorique au XIX ${ }^{\mathrm{e}}$ siècle ", Revue d'histoire littéraire de la France, 80 ${ }^{\mathrm{e}}$ année, $\mathrm{n}^{\circ}$ 2, La Rhétorique au XIX ${ }^{e}$ siècle, mars-avr 1980, p. 181-194.

13. Cf. saint Augustin, Sur la nécessaire rhétorique religieuse.

14. Ferdinand Brunetière, Essais sur la littérature contemporaine, Paris, Calmann-Lévy, 1892, p. 287.

15. Ibid., p. 292.

16. Antoine Albalat, La Formation du style par l'assimilation des auteurs, $2^{\mathrm{e}}$ éd., Paris, Armand Colin, 1902, p. 299.

17. Jules Simon, alors ministre de l'Instruction publique et des Cultes, annonce par une circulaire les prémisses de la réforme de l'enseignement secondaire à venir, par la «[transformation] des 
méthodes qui ont vieilli et [l'abandon] des exercices dont l'inutilité est universellement reconnue » (cf. Antoine Compagnon, op. cit., p. 1216).

18. Instructions concernant le programme de l'enseignement secondaire, 1890, p. 449.

19. Cet aspect est du reste le plus connu, ne serait-ce que par les travaux d'Antoine Compagnon. Cf. La Troisième République des Lettres : de Flaubert à Proust, Paris, Seuil, 1983.

20. Gustave Lanson, «Contre la rhétorique et les mauvaises humanités », dans L'Université et la société moderne, Paris, Armand Colin, 1902, p. 95.

21. Ibid., p. 102.

22. Ibid.

23. Ibid., p. IX.

24. Gustave Lanson, «L'esprit scientifique et la méthode de l'histoire littéraire », conférence faite à l'Université de Bruxelles en 1909, dans Méthodes de l'histoire littéraire, Paris, Belles Lettres, 1925, p. 37.

25. Ibid., p. 24.

26. Ibid., p. 25.

27. Ibid., p. 34.

28. Selon la formule de Barthes, et telle qu'elle sera mise en œuvre dans les années 1950.

29. Dès 1830, Jean-Jacques Ampère cherche à jeter les bases d'une nouvelle critique comme science de la littérature : "Qu'est-ce que la littérature, Messieurs? Ou la littérature est une déclamation vaine, ou elle est une science ; si elle est une science, elle est ou de la philosophie ou de l'histoire. Philosophie de la littérature, Histoire de la littérature, telles sont les deux parties de la science littéraire ; hors de là, je ne vois que les minuties de la critique de détail, ou l'étalage des lieux communs. " (De l'histoire de la poésie : discours prononcé à l'Athénée de Marseille, pour l'ouverture du cours de littérature, le 12 mars 1830, Marseille, Feissat aîné et Demonchy, 1830, p. 7.)

30. Jacky Martin, «Science et rhétorique: la double articulation rhétorique du discours scientifique ", ASp [En ligne], 19-22, 1998, mis en ligne le 30 janvier 2011, p. 2. URL : http:// asp.revues.org/2661.

31. Georg W. Fr. Hegel, Cours d'esthétique, trad. en partie par Ch. Bénard, 4 vol. , Paris/Nancy, 1840-1851 ; Friedrich W. J. Schelling, Écrits philosophiques et morceaux propres à donner une idée générale de son système, trad. par Ch. Bénard, Paris, 1847.

32. Emmanuel Kant, Critique du jugement, suivie des Observations sur le sentiment du beau et du sublime, trad. par J.-R. Barni, 2 vol. , Paris, 1846.

33. A. G. Baumgarten, Æsthetica, Francfort, Oder, 1750. À propos de l'esthétique chez Baumgarten, nous renvoyons à l'article de Syliane Malinowski-Charles, "Goût et jugement des sens chez Baumgarten », Revue germanique internationale [En ligne], 4, 2006, URL : http://rgi.revues.org/142. Pour une histoire allemande du concept d'Ästhetik, voir Joachim Ritter, "Ästhetik, ästhetisch », dans Historisches Wörterbuch der Philosophie, Joachim Ritter, Gottfried Gabriel, Karlfried Gründer (dir.), vol. I, Bâle/Stuttgart, 1971, p.556-580; et pour un aperçu plus général de l'évolution philosophique du concept juqu'au XIX ${ }^{\mathrm{e}}$ siècle, voir l'entrée «Esthétique » du Grand Dictionnaire, op. cit., et l'article d'Herman Parret, « De Baumgarten à Kant : sur la beauté », Revue Philosophique de Louvain, $4^{\mathrm{e}}$ série, t. XC, $\mathrm{n}^{\circ} 87$, 1992, p. 317-343, URL: http://www.persee.fr/doc/ phlou_0035-3841_1992_num_90_87_6745.

34. Charles Lévêque, La Science du beau étudiée dans ses principes, dans ses applications et dans son histoire, Paris, Durand, 1861. Notons que le texte remporte dès 1859 la question mise au concours de l'Académie des sciences morales et politiques sous l'impulsion de Cousin : « Rechercher quels sont les principes de la science du Beau, et les vérifier en les appliquant aux beautés les plus certaines de la nature, de la poésie et des arts, ainsi que par un examen critique des plus célèbres systèmes auxquels cette science du Beau a donné naissance dans l'Antiquité et surtout chez les modernes. » Cf. les «Extraits du rapport» de Jules Barthélémy-Saint-Hilaire présentés en Appendice, $i b i d$. 
35. Anatole France, La Vie littéraire : quatrième série, Paris, Calmann-Lévy, 1888, p. V.

36. Ferdinand Brunetière, L'Évolution des genres dans l'histoire de la littérature, Introduction: L'évolution de la critique depuis la Renaissance jusqu'à nos jours [1890], 6éd., Hachette, Paris, 1914, p. 265-266.

37. Ernest Renan, L'Avenir de la science : pensées de 1848, Paris, Calmann-Lévy, 1890, p. 534.

38. Jean-Jacques Ampère, De l'histoire de la poésie, op. cit., p. 45.

39. Wolf Lepenies, Sainte-Beuve : au seuil de la modernité, Paris, Gallimard, 2002, p. 439-443.

40. Ferdinand Brunetière, Essais sur la littérature contemporaine [1892], $3^{\mathrm{e}}$ éd., Paris, Calmann-Lévy, 1896, p. 12.

41. Ferdinand Brunetière, Manuel de l'histoire de la littérature française, Paris, Delagrave, 1898, p. 468-469.

42. Ferdinand Brunetière, L'Évolution des genres..., op. cit., p. 237.

43. Jacques Fontanille, Sémiotique du discours, Limoges, Presses Universitaires de Limoges, 1998, p. 185.

44. Jean-Michel Berthelot, «Le texte scientifique. Structures et métamorphoses », dans Figures du texte scientifique, PUF, 2003, p. 19-53, ici p. 49.

45. Charles-Augustin Sainte-Beuve, "La Poésie" [lundi 4 janvier1869], dans Nouveaux Lundis, t. XII, 6 éd. revue, Paris, Calmann-Lévy, 1884, p. 4.

46. Charles-Augustin Sainte-Beuve, "Histoire de la littérature anglaise par M. Taine » [Lundi 30 mai1864], dans Nouveaux Lundis, t. VIII, $6^{\mathrm{e}}$ éd. revue, Paris, Calmann-Lévy, 1885, p. 87-88.

47. Ferdinand Brunetière, L'Évolution des genres..., op. cit.

48. Voir notre article dédié à la réception de Claude Bernard par les critiques littéraires: «L'impact de la physiologie dans la critique littéraire de la fin du XIX siècle : l'exemple de Claude Bernard", Épistémocritique, 2018. URL : https://epistemocritique.org/limpact-de-physiologiecritique-litteraire-de-fin-xixeme-siecle-lexemple-de-claude-bernard/

49. Ferdinand Brunetière, Études critiques sur l'histoire de la littérature française [1891], t. IV , $6^{\mathrm{e}}$ éd., Paris, Hachette, 1911, p. 287.

50. Georges Renard, La Méthode scientifique de l'histoire littéraire, Paris, Alcan, 1900, p. 1.

51. Ce réinvestissement est corollaire d'une esthétique moderne qui change de paradigme et qui, à partir de Kant surtout, trouve ses racines dans la subjectivité inhérente à l'homme. Daniel Dumouchel en propose une étude détaillée dans Kant et la genèse de la subjectivité esthétique, Paris, Vrin, 1999. "À l'intérieur de la théorie du jugement esthétique, certaines des "tensions“ constitutives de l'esthétique moderne vont ainsi être théoriquement accueillies : le génie et le goût, l'individu et le sujet, la liberté et la Loi, l'art et la nature, le beau et le sublime, etc. Si aujourd'hui encore nous lisons les remarques de Kant sur l'esthétique avec intérêt, c'est que nous y trouvons, sous une forme largement unifiée, l'incarnation la moins imparfaite de ce que l'on pourrait appeler l'“idéal-type" de la modernité esthétique, en tant qu'“esthétique de la subjectivité".» (p. 7)

52. William Marx, L'Adieu à la littérature. Histoire d'une dévalorisation $\mathrm{XVIII}^{e}-\mathrm{XX} \mathrm{X}^{e}$ siècle, Paris, Minuit, 2005, p. 73. Dans cet essai, Marx identifie ce mouvement du XIX siècle comme allant d'une revalorisation à une « survalorisation de la littérature ».

53. Michel Foucault, Les Mots et les Choses, Paris, Gallimard, « Tel », 1966, p. 312-313. 


\section{RÉSUMÉS}

En faisant le procès de la rhétorique, une partie de la critique littéraire de la seconde moitié du $\mathrm{XIX}^{\mathrm{e}}$ siècle considère l'écriture scientifique comme un nouveau modèle à suivre. Cet article entend resituer le rôle tenu par le discours scientifique, en particulier celui des sciences du vivant, dans le débat sur la rhétorique qui oppose les critiques littéraires de l'époque. Derrière l'ambition des critiques à faire savoir, c'est la valorisation d'une nouvelle idée de la littérature qui nous semble être mise en jeu.

During the second half of the 19th century, part of the literary criticism put rhetoric on trial and praised scientific writing as a new model. This article aims to situate the role played by scientific discourse, especially that of the life sciences, in the debate on rhetoric between the literary critics of the time. Behind the shaping of a discourse that produces knowledge, it is the valorisation of a new idea of literature that seems to be at stake.

INDEX

Keywords : literary criticism, epistemology, rhetoric, aesthetics, discourse

Mots-clés : critique littéraire, épistémologie, rhétorique, esthétique, discours

\section{AUTEUR}

MARINE RIGUET

Université Reims Champagne-Ardenne 